\title{
Effects of tadalafil once daily or on demand versus placebo on time to recovery of erectile function in patients after bilateral nerve-sparing radical prostatectomy
}

\author{
Ignacio Moncada • Fermín R. de Bethencourt • Enrique Lledó-García • \\ Javier Romero-Otero • Carmen Turbi · Hartwig Büttner · Carsten Henneges • \\ Juan I. Martinez Salamanca
}

Received: 31 March 2014 / Accepted: 5 August 2014 / Published online: 26 August 2014

(C) The Author(s) 2014. This article is published with open access at Springerlink.com

\begin{abstract}
Purpose We report time to erectile function (EF)-recovery data from a multicenter, randomized, double-blind, double-dummy, placebo-controlled trial evaluating tadalafil started after bilateral nerve-sparing radical prostatectomy (nsRP).

Methods Patients $\leq 68$ years were randomized post-nsRP 1:1:1 to 9-month double-blind treatment (DBT) with tadalafil $5 \mathrm{mg}$ once daily $(\mathrm{OaD}), 20 \mathrm{mg}$ tadalafil on demand ("pro-re-nata"; PRN), or placebo, followed by 6-week drug-free washout (DFW) and 3-month open-label $\mathrm{OaD}$
\end{abstract}

Electronic supplementary material The online version of this article (doi:10.1007/s00345-014-1377-3) contains supplementary material, which is available to authorized users.

I. Moncada $(\bowtie)$

Department of Urology, Hospital La Zarzuela, Madrid, Spain

e-mail: ignacio@moncada.name

F. R. de Bethencourt

Department of Urology, Hospital “La Paz”, Madrid, Spain

E. Lledó-García

Departament of Urology, Hospital General Universitario

Gregorio Marañón, Madrid, Spain

J. Romero-Otero

Department of Urology, Hospital Universitario 12 Octubre,

Madrid, Spain

C. Turbi

Eli Lilly and Company, Madrid, Spain

H. Büttner · C. Henneges

Lilly Deutschland GmbH, Bad Homburg, Germany

J. I. Martinez Salamanca

Department of Urology, Hospital Universitario Puerta de

Hierro-Majadahonda, Madrid, Spain treatment. Secondary outcome measures included KaplanMeier estimates of time to EF-recovery (IIEF-EF $\geq 22$ ) during DBT (Cox proportional hazard model adjusting for treatment, age, and country).

Results A total of 423 patients were randomized to tadalafil $\mathrm{OaD}(N=139)$, PRN $(N=143)$, and placebo $(N=141) ; 114 / 122 / 155$ completed DBT. The proportion of patients achieving IIEF-EF $\geq 22$ at some point during DBT with $\mathrm{OaD}, \mathrm{PRN}$, and placebo was $29.5,23.9$, and $18.4 \%$, respectively. DBT was too short to achieve EF-recovery (IIEF-EF $\geq 22$ ) in $>50 \%$ of patients; median time to EFrecovery was non-estimable. Time for $25 \%$ of patients to achieve EF-recovery $(95 \% \mathrm{CI})$ was $5.8(4.9,9.2)$ months for $\mathrm{OaD}$ versus $9.0(5.5,9.2)$ and $9.3(9.0,9.9)$ months for PRN and placebo, respectively. Showing a significant overall treatment effect $(p=0.038)$, the probability for EF-recovery was significantly higher for $\mathrm{OaD}$ versus placebo [hazard ratio (HR); $95 \%$ CI 1.9; 1.2, 3.1; $p=0.011$ ], but not for PRN versus placebo $(p=0.140)$. Of $57 \mathrm{OaD}$ patients $(41.0 \%)$ with ED improved (by $\geq 1$ IIEF-EF severity grade) at the end of DBT, 16 (28.1\% of 57) maintained this improvement through DFW and 27 (47.4\%) declined but maintained improvement from baseline after DFW.

Conclusions Data suggest that the use of tadalafil $\mathrm{OaD}$ can significantly shorten the time to EF-recovery post-nsRP compared with placebo.

Keywords Nerve-sparing prostatectomy $\cdot$ Penile function · Phosphodiesterase 5 inhibitors - Prostate cancer . Rehabilitation · Tadalafil once a day
Abbreviations
CI Confidence interval
DBT Double-blind treatment
DFW Drug-free washout 


$\begin{array}{ll}\text { ED } & \text { Erectile dysfunction } \\ \text { EF } & \text { Erectile function } \\ \text { HR } & \text { Hazard ratio } \\ \text { IIEF-EF } & \begin{array}{l}\text { International Index of Erectile Function-Erec- } \\ \text { tile Function domain score }\end{array} \\ \text { ITT } & \text { Intent-to-treat } \\ \text { LSmeans } & \text { Least square means } \\ \text { MCID } & \text { Minimally clinically important difference } \\ \text { MMRM } & \text { Mixed model for repeated measures } \\ \text { nsRP } & \text { Nerve-sparing radical prostatectomy } \\ \text { OaD } & \text { Once daily } \\ \text { OLT } & \text { Open-label OaD treatment } \\ \text { PDE5 } & \text { Phosphodiesterase type 5 } \\ \text { PRN } & \text { "Pro-re-nata," on demand } \\ \text { RCTs } & \text { Randomized clinical trails } \\ \text { REF } & \text { Residual erection function } \\ \text { SD } & \text { Standard deviation }\end{array}$

\section{Introduction}

Erectile dysfunction (ED) can be a relatively common sequela after radical prostatectomy for localized prostate cancer [1-3], despite the use of nerve-sparing techniques (nsRP). Many men may not recover erectile function (EF) for more than 18 months post-nsRP [4, 5]. Rarely, time to EF-recovery can extend well beyond 2 years [6]. The rate of and time to EF-recovery post-nsRP can vary widely and may be influenced by a number of factors, including patient age, type of surgery, and treatment during the recovery phase [5-7].

Phosphodiesterase type 5 (PDE5)-inhibitors are generally well-tolerated and effective in the treatment of ED post-nsRP $[1,2,8]$. However, they are less effective in the post-nsRP population when compared to the general population, and the optimal time point for starting PDE5-inhibitor treatment is still undetermined [9].

To date, four randomized clinical trials (RCTs) have evaluated the impact of the early use of short-acting PDE5inhibitors on EF-recovery in men post-nsRP. Sildenafil, vardenafil, and avanafil have all been shown to improve drug-assisted EF when given on demand ("pro-re-nata," PRN) [10-12]. However, EF-recovery up to 1 year postnsRP did not differ between sildenafil given once daily $(\mathrm{OaD})$ or PRN [13].

For the long-acting PDE5-inhibitor tadalafil, an initial retrospective study in 92 patients showed that tadalafil $\mathrm{OaD}$ started early after robot-assisted laparoscopic nsRP was well tolerated and significantly improved EF compared to patients without PDE5-inhibitor treatment [14].

Montorsi et al. [15] have published a randomized, placebo-controlled trial evaluating the early use of tadalafil, given $\mathrm{OaD}$ or PRN, on both drug-assisted EF after
9 months of double-blind treatment (DBT) with tadalafil $\mathrm{OaD}$ or PRN and on unassisted EF (without PDE5-inhibitor support) after 6 weeks of drug-free washout (DFW) in men who developed ED post-nsRP. Tadalafil OaD improved drug-assisted EF-recovery, as measured by the proportion of patients achieving an International Index of Erectile Function-Erectile Function domain score (IIEF-EF) $\geq 22$ at the end of DBT, while unassisted EF-recovery after DFW was not improved by tadalafil OaD or PRN [15].

This paper specifically addresses the effects of tadalafil OaD and PRN treatment on the time to recovery of EF (IIEF-EF $\geq 22$ ) during the DBT period of this trial and the maintenance of treatment response after DFW.

\section{Materials and methods}

Patients

Adult men aged $<68$ years at the time of nsRP with normal preoperative $\mathrm{EF}$ who underwent nsRP for organ-confined, non-metastatic prostate cancer (Gleason score $\leq 7$, prostate specific antigen $<10 \mathrm{ng} / \mathrm{mL}$ ) were enrolled between November 2009 and August 2011 in 50 centers from nine European countries and Canada (NCT01026818). Post-surgical inclusion criteria included the development of ED, as measured by a patient-reported Residual Erection Function (REF) score of $\leq 3$ ("penis is hard enough for penetration but not completely hard"). This criterion was used because of the limited validity of IIEF-EF domain scores for direct EF assessment post-nsRP [15, 16]. Detailed eligibility criteria have been published [16].

\section{Trial design}

This multicenter, Phase IV, randomized, double-blind, 3-arm, placebo-controlled parallel-group trial consisted of the following periods, as previously described (Supplementary Figure S1) [15]: screening (including nsRP surgery), 9-month randomized, double-blind, double-dummy treatment with $5 \mathrm{mg}$ tadalafil $\mathrm{OaD}, 20 \mathrm{mg}$ tadalafil PRN, or placebo (DBT); 6-week DFW; and 3-month open-label treatment with $5 \mathrm{mg} /$ day tadalafil OaD (OLT, all patients).

\section{Outcome measures}

\section{IIEF-EF scores}

The primary objective was to evaluate the efficacy of tadalafil $\mathrm{OaD}$ and tadalafil PRN, compared with placebo, in improving unassisted EF (EF after 6 weeks of DFW), as measured by the proportion of patients achieving an IIEFEF score $\geq 22$ at the end of DFW (primary outcome) [15]. 
An IIEF-EF $\geq 22$ was required at screening (after cancer diagnosis, $\leq 6$ weeks pre-nsRP). This cutoff was considered appropriate because many men with newly diagnosed prostate cancer claim to have unimpaired EF, but have IIEF-EF scores of 22-25 (mild ED) [15, 17]. Time to EF-recovery (additional secondary analysis pre-specified in the statistical analysis plan, finalized and approved before database lock) was defined as the time from baseline to reach an IIEF-EF $\geq 22$ during DBT.

\section{ED severity}

IIEF-EF scores were categorized into the following ED severity categories: severe (0-10), moderate (11-16), mild (17-25), and normal (26-30) [18]. ED severity was assessed at baseline, end of DBT, and end of DFW. Improvement was defined as an IIEF-EF score of $\geq 1$ category higher than baseline (or maintaining normal $\mathrm{EF}$ ) at the end of DBT. Maintenance of treatment response, assessed for patients who improved $\geq 1$ category after DBT, was defined as either maintaining this improved category until the end of DFW or declining after DBT but still maintaining a higher category at the end of DFW than at baseline.

\section{Statistical analysis}

The planned sample size of 412 patients was based on the primary outcome (proportion of patients achieving IIEF-EF 222) [15]. All analyses were based on the intent-to-treat (ITT) population, including all randomized patients with baseline data and at least one post-baseline visit. Pre-specified treatment group comparisons were tadalafil $\mathrm{OaD}$ versus placebo and tadalafil PRN versus placebo.

The Kaplan-Meier product-limit method was used to estimate rates for the time to EF-recovery (IIEF-EF $\geq 22$ ) including $95 \%$ confidence intervals (CI). Only patients with IIEF-EF $<22$ at screening were included; patients not reaching IIEF-EF $\geq 22$ were censored at the end of DBT. Hazard ratios (HRs) and $p$ values were derived from a Cox proportional hazard model adjusting for treatment, age ( $<61$ years vs. 61-68 years), and country.

IIEF-EF score changes from baseline were analyzed using a mixed model for repeated measures (MMRM) analysis, assuming an unstructured covariance structure and including visit, treatment, treatment-by-visit interaction, country, age group, and baseline as fixed effects, and patient and error as random effects. Adjusted least square means (LSmeans) and $95 \%$ CIs were calculated from the model. A minimally clinically important difference (MCID), defined as $\geq 4$ points difference in IIEF-EF [19], was used to determine the average needed treatment effect that has clinical relevance for patients.
For $p$ values, a $5 \%$ level of significance was used. Data were analyzed using the SAS 9.2 software (SAS Institute Inc., Cary, USA).

\section{Results}

Patient disposition and baseline characteristics

Of 583 patients screened, 423 were randomized: 139 (32.9\%) to tadalafil OaD, 143 (33.8\%) to tadalafil PRN, and $141(33.3 \%)$ to placebo (Supplementary Figure S2). Patients in the PRN group took a mean (SD) of 1.5 (0.95) tadalafil $20 \mathrm{mg}$ tablets per week. Patient disposition, baseline demographics, and relevant disease characteristics were balanced in all 3 treatment groups (Table 1) [15]. As per inclusion criteria, all patients had to have IIEF-EF $\geq 22$ pre-nsRP. Post-nsRP at baseline, $83.9 \%$ of patients reported severe ED based on IIEF-EF scores (mean [standard deviation; SD] score 6.4 [5.81]) and $>98 \%$ reported an $\mathrm{REF} \leq 3$.

\section{Time to EF-recovery during DBT}

The proportion of patients achieving IIEF-EF $\geq 22$ at any time point during DBT with $\mathrm{OaD}$, PRN, and placebo was 29.5, 23.9, and $18.4 \%$, respectively. Based on the KaplanMeier analysis, $25 \%$ of patients achieved EF-recovery (IIEF-EF $\geq 22$ ) within 5.8 months for tadalafil $\mathrm{OaD}$, 9.0 months for tadalafil PRN, and 9.3 months for placebo (Fig. 1a). Median time to EF-recovery could not be estimated as $<50 \%$ of patients achieved EF-recovery during the 9-month DBT period (Supplementary Figure S3). The Cox proportional hazard model showed a significant overall treatment effect $(p=0.038)$. Patients in the tadalafil OaD (but not PRN) group had a significantly higher probability for EF-recovery versus placebo (HR [95\% CI]: tadalafil $\mathrm{OaD}$ versus placebo: $1.90[1.16,3.12], p=0.011$; tadalafil PRN versus placebo: 1.47 [0.88, 2.47], $p=0.140$ ). Age group had no significant effect on time to EF-recovery $(p=0.223$; Supplementary Figure S4).

\section{LSmean IIEF-EF improvements}

LSmean IIEF-EF improvement during DBT significantly exceeded the MCID (Lower $95 \%$ CI LSmean $\triangle$ IIEF$\mathrm{EF} \geq 4$ ) at month 5 in the tadalafil $\mathrm{OaD}$ treatment group (LSmean [95 \% CI]: $6.9[5.0,8.8]$ ) and month 9 in the tadalafil PRN treatment group $(6.5$ [4.6, 8.5]) (Fig. 2). For placebo, LSmean IIEF-EF did not significantly exceed the MCID before month 10.5 (end of DFW: 6.0 [3.9, 8.0]). The treatment effect versus placebo was statistically significant 
Table 1 Baseline

characteristics and status post nsRP
$B M I$ body mass index, $E D$ erectile dysfunction, IIEF$E F$ International Index of Erectile Function-Erectile Function, $N$ total number of patients, $n$ number of patients, $n s R P$ bilateral nerve-sparing prostatectomy, $O a D$ once a day, $P R N$ "pro-re-nata"/on demand, $R E F$ residual erectile function, $S D$ standard deviation

${ }^{\text {a }}$ Based on intent-to-treat population, excluding one patient from the tadalafil PRN group with no post-baseline data

b Two patients in the tadalafil $\mathrm{OaD}$ group and two patients in the tadalafil PRN group had missing values

\begin{tabular}{|c|c|c|c|}
\hline Variable & Tadalafil OaD $(N=139)$ & Tadalafil PRN $(N=143)$ & Placebo $(N=141)$ \\
\hline \multicolumn{4}{|l|}{ Age (years) } \\
\hline Mean (SD) & $58.6(5.07)$ & $57.5(5.91)$ & $57.6(5.69)$ \\
\hline$<61(n, \%)$ & $82(59.0)$ & $85(59.4)$ & $91(64.5)$ \\
\hline $61-68(n, \%)$ & $57(41.0)$ & $58(40.6)$ & $50(35.5)$ \\
\hline \multicolumn{4}{|l|}{ Ethnicity $(n, \%)$} \\
\hline Caucasian & 137 (98.6) & $141(98.6)$ & $138(97.9)$ \\
\hline \multicolumn{4}{|l|}{$B M I\left(\mathrm{~kg} / \mathrm{m}^{2}\right)$} \\
\hline Mean (SD) & $26.6(2.97)$ & $26.9(2.93)$ & $27.1(3.08)$ \\
\hline \multicolumn{4}{|l|}{$I I E F-E F$} \\
\hline$N$ with data & 137 & 140 & 137 \\
\hline Mean (SD) & $6.0(5.80)$ & $6.7(5.57)$ & $6.5(6.08)$ \\
\hline \multicolumn{4}{|c|}{ ED severity (IIEF-EF categories) $(n, \%)^{\mathrm{a}}$} \\
\hline Missing & $2(1.4)$ & $2(1.4)$ & $4(2.8)$ \\
\hline Normal (26-30) & $4(2.9)$ & $2(1.4)$ & $2(1.4)$ \\
\hline Mild (17-25) & $5(3.6)$ & $8(5.6)$ & $9(6.4)$ \\
\hline Moderate (11-16) & $9(6.5)$ & $10(7.0)$ & $11(7.8)$ \\
\hline Severe $(0-10)$ & $119(85.6)$ & $120(84.5)$ & $115(81.6)$ \\
\hline $\mathrm{REF} \leq 3^{\mathrm{b}}$ & $137(98.6)$ & $138(97.2)$ & $141(100)$ \\
\hline \multicolumn{4}{|l|}{$n s R P$ approach $(n, \%)$} \\
\hline Open surgery & $68(48.9)$ & $65(45.5)$ & $56(39.7)$ \\
\hline Conventional laparoscopy & $29(20.9)$ & $31(21.7)$ & $28(19.9)$ \\
\hline Robot-assisted laparoscopy & $31(22.3)$ & $41(28.7)$ & $44(31.2)$ \\
\hline Other & $11(7.9)$ & $6(4.2)$ & $13(9.2)$ \\
\hline \multicolumn{4}{|c|}{ Total nerve-sparing score post-nsRP $(n, \%)$} \\
\hline Perfect (2) & $117(84.2)$ & $116(81.1)$ & $113(80.1)$ \\
\hline Not perfect $(>2)$ & $22(15.8)$ & 27 (18.9) & $28(19.9)$ \\
\hline
\end{tabular}

for tadalafil OaD only (LSmean difference [95 \% CI]: 2.8 $[0.8,4.8] ; p=0.007)$ at month 9 .

ED severity improvement and maintenance of improvement

At baseline (randomization), $83.9 \%$ of patients overall had severe ED (IIEF-EF 0-10; Table 1). During DBT, improvement in ED severity by $\geq 1$ severity grade was achieved by $41.0 \%$ of all tadalafil OaD patients, $38.7 \%$ of all tadalafil PRN patients, and $22.7 \%$ of patients on placebo (Fig. 2). In all groups, the majority of those patients who had improved during DBT maintained an improvement of $\geq 1$ severity grade from baseline through DFW (improvement maintained, Fig. 2). For tadalafil OaD (Fig. 2), 43 of 57 improved patients (75.4\%) were still improved from baseline after DFW, including 16 patients $(28.1 \%)$ who maintained the improvement they had reached at the end of DBT and 27 patients $(47.4 \%)$ who declined but still maintained improvement from baseline after DFW.

\section{Discussion}

This trial was the first RCT in patients with established ED post-nsRP which investigated the effect of early treatment with tadalafil $\mathrm{OaD}$ and PRN on EF-recovery. As previously reported, tadalafil OaD significantly improved drug-assisted EF-recovery, as measured by the proportion of patients achieving IIEF-EF $\geq 22$ at the end of DBT $(25.2 \%$ of OaD patients versus $14.2 \%$ in placebo group; $p=0.016$; Supplementary Figure S3). Unassisted EF-recovery after DFW was not improved by tadalafil OaD or PRN [15]. Here, we show that tadalafil OaD (but not PRN) significantly shortened the time to EF-recovery during DBT when compared with placebo: with placebo, it took 9.3 months until $25 \%$ of patients had reached EF-recovery; this period was shortened by 3.5 months (i.e., to 5.8 months) in the tadalafil OaD treatment group. An early start of penile rehabilitation does seem to be important; Mulhall et al. [20] showed that patients who started PDE5-inhibitor treatment early post-nsRP reached significantly higher mean IIEF-EF scores than patients who started later at $\geq 6$ months post-nsRP $(p<0.0001)$. 
(a)
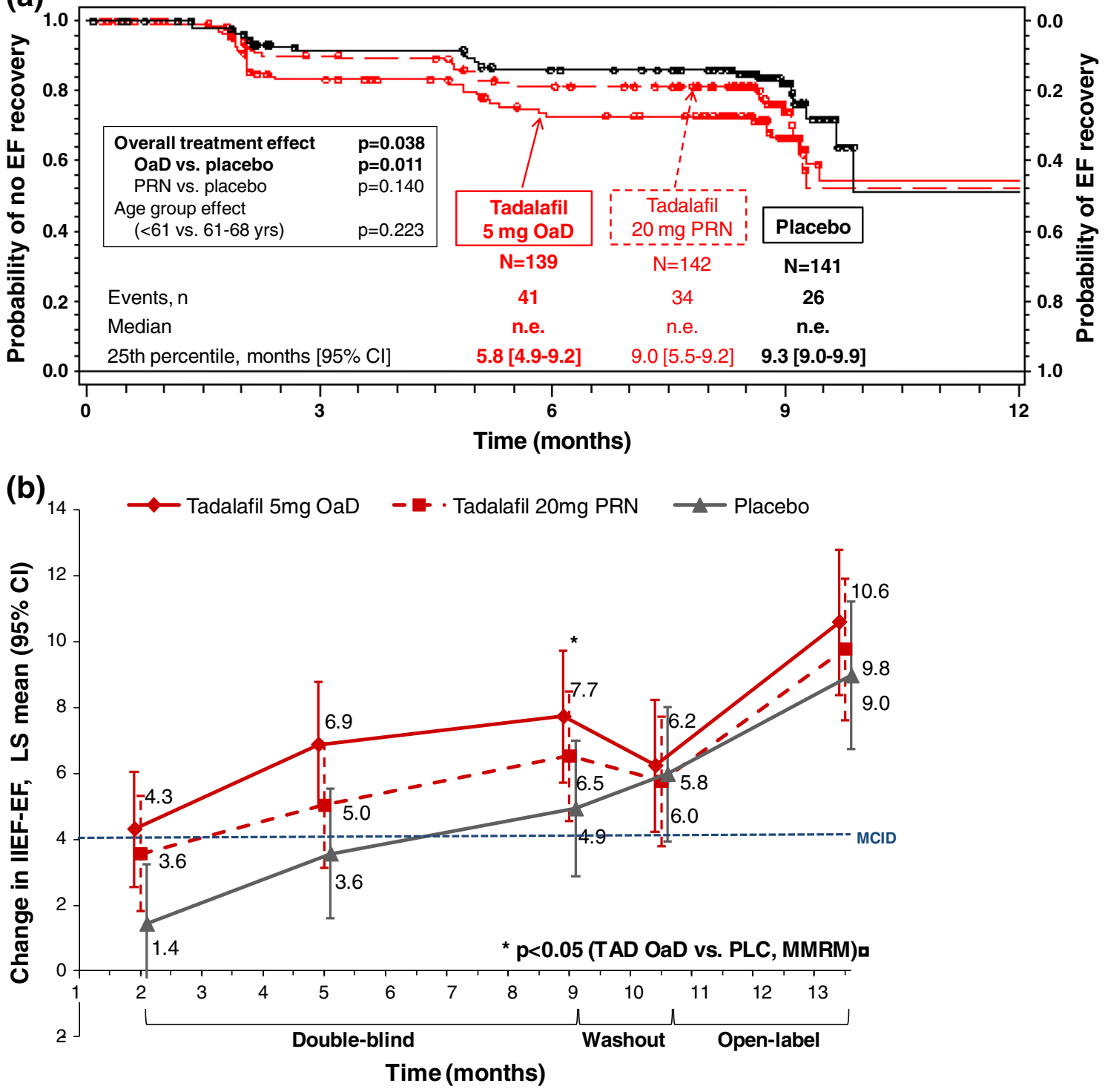

Fig. 1 EF-recovery (IIEF-EF $\geq 22$ ) and EF category improvement during DBT. $C I$ confidence interval, $D B T$ double-blind treatment, $E F$ erectile function, IIEF-EF International Index of Erectile FunctionErectile Function domain, LSmean least square mean, MCID minimal clinically important difference, $M M R M$ mixed model for repeated measures, $n$ number of events, $N$ number of patients, n.e. not estimable, n.s. not significant, $O a D$ once a day, $P L C$ placebo, $P R N$ "pro-renata"/on demand, TAD tadalafil, yrs years. a Event (EF-recovery) was

EF-recovery rates during 9-month DBT were $<50 \%$ in all treatment groups, which is in line with the published data on natural EF-recovery post-nsRP. Without treatment, time to EF-recovery averages 18 months [4] and can extend well beyond 2 years [5, 6]. In a study by Gallina et al. [21], only $35.8 \%$ of untreated patients reached EF-recovery (IIEF-EF $\geq 22$ ) after an average of 26.8 months post-nsRP.

However, LSmean IIEF-EF improvement in the tadalafil OaD group significantly exceeded the MCID defined as change in IIEF-EF from $<22$ at screening to $\geq 22$. $P$ values are obtained from Cox proportional hazard model including terms for treatment, country, and age. b $p$ value obtained from an MMRM model, assuming an unstructured covariance structure, including terms for visit, treatment, treatment-by-visit interaction, country, age group, and baseline IIEF-EF score as fixed effects, and patient and error as random effects. Previously published in: Montorsi et al. 2014 [15]

$(\Delta \mathrm{IIEF-EF} \geq 4[19])$ already at month 5 of DBT, as compared to month 9 for tadalafil PRN; improvement with placebo did not significantly exceed the MCID before month 10.5 (end of DFW). At the end of DBT, the treatment effect versus placebo was statistically significant for tadalafil OaD only $(p=0.007)$.

Chronic (daily) dosing of tadalafil, but not PRN treatment, will lead to steady state PDE5-inhibition [22] which may be associated with prolonged (continuous) periods 


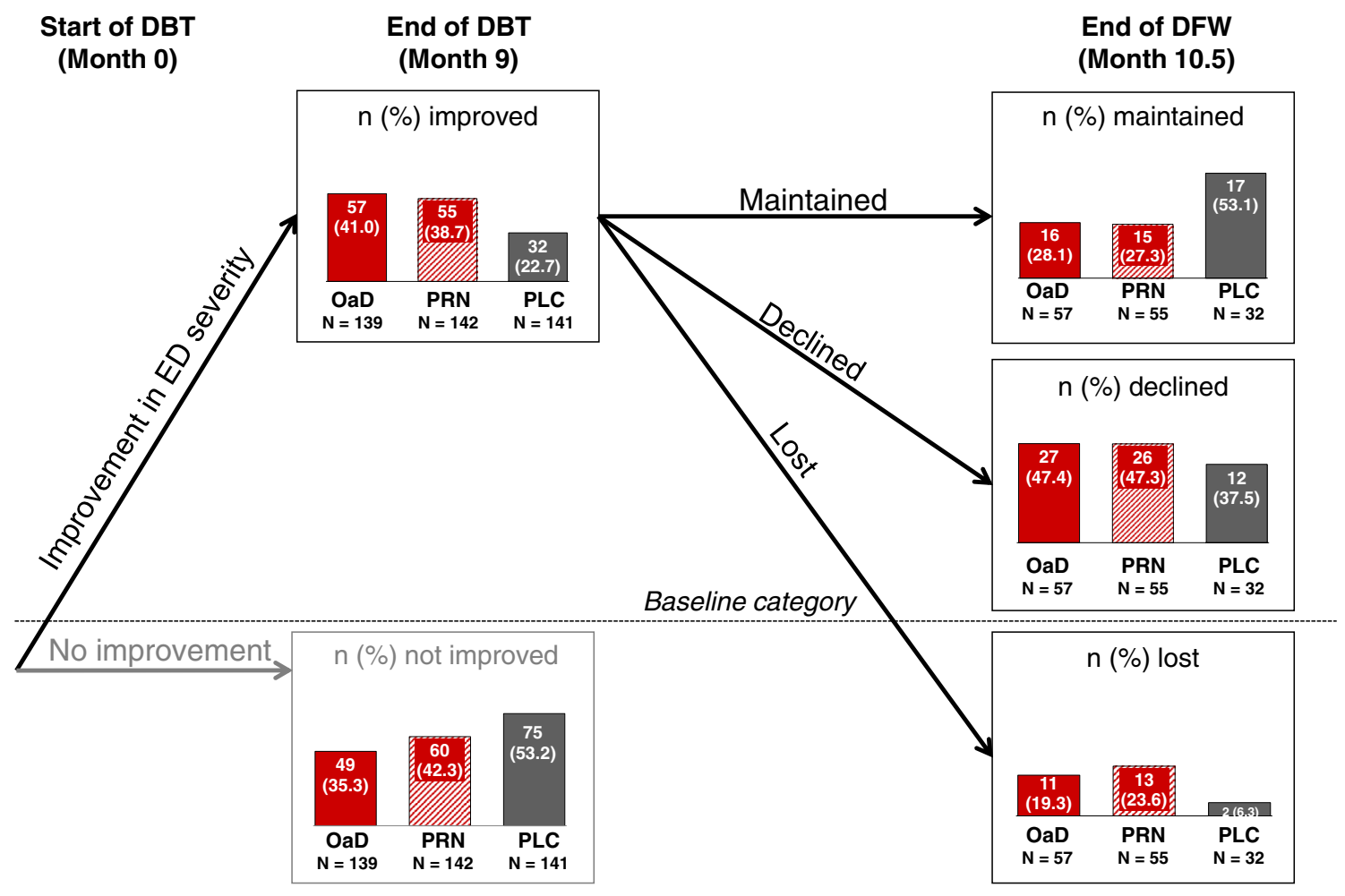

Fig. 2 Improvement and maintenance of improvement from baseline in ED severity (based on IIEF-EF categories). $D B T$ double-blind treatment, $D F W$ drug-free washout, $E D$ erectile dysfunction, $I I E F-E F$ International Index of Erectile Function-Erectile function domain, $O a D$ once a day, $P L C$, placebo, $P R N$ "pro-re-nata"/on demand, $N$ total number of patients, $n$ number of patients. IIEF-EF scores defining ED severity categories: severe, 0-10; moderate, 11-16; mild, 1725; normal, 26-30. Improvement was defined as reporting an IIEFEF score of at least 1 category higher than baseline (or maintaining normal EF). Improvement declined was defined as reporting IIEF-EF scores at the end of DFW that were less than the end of the DBT but still at least 1 category higher than baseline. Improvement maintained was defined as reporting IIEF-EF scores at the end of DFW that were

of increased tissue oxygenation during the post-operative regenerative process. Preclinical data suggest that chronic low-dose administration may protect from structural changes of penile cavernous corpora and is associated with EF enhancement [23-25].

None of the other RCTs on PDE5-inhibitors post-nsRP has reported time to EF-recovery data based on KaplanMeier analysis. However, data from 2 non-RCTs indicated that PDE5-inhibitor treatment may shorten time to EFrecovery [26, 27]. Bannowsky et al. [26] reported a significant difference in time to EF-recovery between patients receiving nightly low-dose sildenafil for up to 12 months when compared with patients receiving no treatment $(p<0.001)$. In agreement with these results, Briganti et al. showed that patients receiving any PDE5-inhibitor $(\mathrm{OaD}$ or PRN) achieved significantly higher 3-year EF-recovery rates (IIEF-EF $\geq 22$ ) than patients receiving placebo (72 vs. at least as high or higher than scores at the end of DBT. Improvement lost was defined as IIEF-EF scores that were less than or equivalent to ED severity at baseline. All percentage are relative to the size of each treatment group ("N," provided below each bar) not relative to the overall population. Missing data: For 33 patients $(23.7 \%)$ in the tadalafil OaD group, for $27(19.0 \%)$ in the tadalafil PRN group, and for $34(24.1 \%)$ in the placebo group, improvement could not be calculated because the patient either discontinued during DBT or had missing IIEF-EF scores at baseline and/or month 9. For three patients $(5.3 \%)$ in the tadalafil $\mathrm{OaD}$ group, one $(1.8 \%)$ in the tadalafil PRN group, and one $(3.1 \%)$ in the placebo group, maintenance of improvement could not be calculated because the patients either discontinued during DFW or had missing IIEF-EF scores at month 10.5

$38 \%, p \leq 0.001$, Kaplan-Meier analysis). For the overall population studied, no significant difference was observed between $\mathrm{OaD}$ and PRN treatment. However, patients with an intermediate risk of ED (66-69 years or IIEF-EF 11-25, and Charlson Comorbidity Index $\leq 1$ ), who shared key criteria with our patient population of low/intermediate ED risk (average patient $\leq 61$ years; IIEF-EF $\geq 22$ at baseline), achieved significantly higher 3-year EF-recovery rates with OaD compared to PRN treatment (74 vs. $52 \% ; p=0.02$ ) [27]. As suggested by Castiglione et al. [28], the effect of PDE5-inhibitor treatment post-nsRP may be maximal in patients with intermediate ED risk. To date, trials have focused on populations with low ED risk [11, 13, 15].

In our trial, age group had no significant effect on the time to EF-recovery during DBT or on the proportion of patients achieving EF-recovery during DBT [15]. However, after DFW (Month 10.5), younger patients ( $<61$ years) 
were significantly more probable to achieve EF-recovery than older patients $(p=0.020 ;[15])$. These results are in line with literature.

A 2010 study by Briganti et al. [29] showed that younger patients ( $\leq 65$ years) were more likely to recover EF (IIEF$\mathrm{EF} \geq 22$ ) than older patients. A meta-analysis by Kilminster et al. and studies by Nelson et al. and Gallina et al. also showed that younger patients were significantly more likely to recover EF post-nsRP than older patients $[5,7,21]$.

A clear limitation of the current trial was that the 9-month DBT phase was too short for full assessment of EF-recovery. We cannot exclude that the treatment effect of tadalafil OaD may be lost by the end of 2 years due to spontaneous EF-recovery in the placebo arm. Valid statistical analysis of time to EF-recovery could not be performed on IIEF-EF data collected after DBT (i.e., after DFW at month 10.5 or OLT at month 13.5) due to the break in ED treatment for 6 weeks. The significant treatment effect on EFrecovery was lost during the DFW. However, after 3-month OLT with tadalafil OaD (month 13.5), the proportion of patients with EF-recovery increased in all treatment groups (32.4\% in the tadalafil OaD group; $33.1 \%$ in the tadalafil PRN group; and $27.0 \%$ in the placebo group) [15]. Further, the results of the Briganti study indicate that the significant effect of $\mathrm{OaD}$ treatment on time to recovery may persist after longer follow-up periods (up to 3 years) [27]. In this context, the demonstrated maintenance of tadalafil's treatment effect could play an important role in future studies that allow for longer treatment or follow-up periods. Even after DFW, over $75 \%$ of patients treated with tadalafil OaD maintained an improvement in ED severity from baseline.

In conclusion, patients taking tadalafil $\mathrm{OaD}$ (but not those taking PRN) significantly shortened the time to EFrecovery during DBT when compared with placebo. No statistically significant difference in time to EF-recovery was observed between younger and older patients. These data suggest that tadalafil $\mathrm{OaD}$, if started early, may accelerate EF-recovery post-nsRP.

Acknowledgments We thank all patients for participating and all trial investigators for their contribution to data acquisition and patient care. We thank Julia Branicka and Tom Clayton, both Eli Lilly and Company, for supporting the conduct of the trial. We thank Joaquin Casariego and Kraig Kinchen, Eli Lilly and Company, for medical advice. We thank Clare Barker, Bruce Basson, Ann Gibb and Pepa Polavieja, all from Eli Lilly and Company, for statistical support. Statistical analyses were programmed by PSI CRO LTD, St. Petersburg, Russia. We thank Shawna Crawford and Karin Helsberg, Trilogy Writing and Consulting GmbH, Frankfurt, Germany, for providing medical writing services on behalf of Eli Lilly.

Conflict of interest The trial has been funded by Eli Lilly and Company. I. Moncada has been a consultant for and received speaker honoraria and travel expenses from Eli Lilly. C. Henneges, C. Turbi and H. Buettner are employees of Eli Lilly and Company and own Eli Lilly stock. F.R. de Bethencourt, E. Lledó-García, J.I
Martinez-Salamanca and J. Romero-Otero have no conflicts of interest to disclose.

Ethical standard The study was conducted in accordance with the ethical standards laid down in the 1964 Declaration of Helsinki and its later amendments; appropriate ethical review boards approved the trial protocol for each country. All patients had signed written informed consent prior to their inclusion in the study.

Open Access This article is distributed under the terms of the Creative Commons Attribution License which permits any use, distribution, and reproduction in any medium, provided the original author(s) and the source are credited.

\section{References}

1. Salonia A, Burnett AL, Graefen M et al (2012) Prevention and management of postprostatectomy sexual dysfunctions. Part 1: choosing the right patient at the right time for the right surgery. Eur Urol 62:261-272

2. Salonia A, Burnett AL, Graefen M et al (2012) Prevention and management of postprostatectomy sexual dysfunctions. Part 2: recovery and preservation of erectile function, sexual desire, and orgasmic function. Eur Urol 62:273-286

3. Ficarra V, Novara G, Ahlering TE et al (2012) Systematic review and meta-analysis of studies reporting potency rates after robotassisted radical prostatectomy. Eur Urol 62:418-430

4. Stanford JL, Feng Z, Hamilton AS et al (2000) Urinary and sexual function after radical prostatectomy for clinically localized prostate cancer: the Prostate Cancer Outcomes Study. JAMA 283:354-360

5. Kilminster S, Müller S, Menon M, Joseph JV, Ralph DJ, Patel HRH (2011) Predicting erectile function outcome in men after radical prostatectomy for prostate cancer. BJU Int 110:422-426

6. Sivarajan G, Prabhu V, Taksler GB, Laze J, Lepor H (2014) Tenyear outcomes of sexual function after radical prostatectomy: results of a prospective longitudinal study. Eur Urol 65:58-65

7. Nelson CJ, Scardino PT, Eastham JA, Mulhall JP (2013) Back to baseline: erectile function recovery after radical prostatectomy from the patients' perspective. J Sex Med 10:1636-1643

8. Montorsi F, Nathan PH, McCullough A et al (2004) Tadalafil in the treatment of erectile dysfunction following bilateral nerve sparing radical retropubic prostatectomy: a randomized, doubleblind, placebo controlled trial. J Urol 172:1036-1041

9. Hatzimouratidis K, Burnett AL, Hatzichristou D, McCullough AR, Montorsi F, Mulhall JP (2009) Phosphodiesterase type 5 inhibitors in postprostatectomy erectile dysfunction: a critical analysis of the basic science rationale and clinical application. Eur Urol 55:334-347

10. Nathan PH, McCullough AR, Levine LA et al (2008) Randomized, double-blind, placebo-controlled study of postoperative nightly sildenafil citrate for the prevention of erectile dysfunction after bilateral nerve-sparing radical prostatectomy. Int J Impot Res 20:479-486

11. Montorsi F, Brock G, Lee J et al (2008) Effect of nightly versus on demand vardenafil on recovery of erectile function in men following bilateral nervesparing radical prostatectomy. Eur Urol 54:924-931

12. Mulhall JP, Burnett AL, Wang R et al (2013) A phase 3, placebo controlled study of the safety and efficacy of avanafil for the treatment of erectile dysfunction after nerve sparing radical prostatectomy. J Urol 189:2229-2236

13. Pavlovich CP, Levinson AW, Su LM et al (2013) Nightly vs ondemand sildenafil for penile rehabilitation after minimally invasive nerve-sparing radical prostatectomy: results of a randomized double-blind trial with placebo. BJU Int 112:844-851 
14. Seo YE, Kim SD, Kim TH, Sung GT (2014) The efficacy and safety of tadalafil $5 \mathrm{mg}$ once daily in the treatment of erectile dysfunction after robot-assisted laparoscopic radical prostatectomy: 1-year follow-up. Korean J Urol 55:112-119

15. Montorsi F, Brock G, Stolzenburg JU et al (2014) Effects of tadalafil treatment on erectile function recovery post bilateral nervesparing radical prostatectomy: a randomized placebo-controlled study. Eur Urol 65:587-596

16. Mulhall JP, Goldstein I, Bushmakin AG et al (2007) Validation of the erection hardness score. J Sex Med 4:1626-1634

17. Cappelleri JC, Rosen RC, Smith MD, Mishra A, Osterloh IH (1999) Diagnostic evaluation of the erectile function domain of the International Index of Erectile Function. Urology 54:346-351

18. Porst H, Glina S, Ralph D, Zeigler H, Wong DG, Woodward B (2010) Durability of response following cessation of tadalafil taken once daily as treatment for erectile dysfunction. J Sex Med 7:3487-3494

19. Rosen RC, Allen KR, Ni X, Araujo AB (2011) Minimal clinically important differences in the erectile function domain of the International Index of Erectile Function scale. Eur Urol 60:1010-1016

20. Mulhall JP, Parker M, Waters BW, Flanigan R (2010) The timing of penile rehabilitation after bilateral nerve-sparing radical prostatectomy affects the recovery of erectile function. BJU Int 105(1):37-41

21. Gallina A, Ferrari M, Suardi N et al (2012) Erectile function outcome after bilateral nerve sparing radical prostatectomy: which patients may be left untreated? J Sex Med 9:903-908

22. Forgue ST, Patterson BE, Bedding AW et al (2006) Tadalafil pharmacokinetics in healthy subjects. Br J Clin Pharmacol 61:280-288
23. Aydogdu O, Gokce MI, Burgu B, Baltacı S, Yaman O (2011) Tadalafil rehabilitation therapy preserves penile size after bilateral nerve sparing radical retropubic prostatectomy. Int Braz J Urol 37:336-346

24. Mostafa ME, Senbel AM, Mostafa T (2013) Effect of chronic low-dose tadalafil on penile cavernous tissues in diabetic rats. Urology 81:1253-1259

25. Park K, Ryu KS, Li WJ, Kim SW, Paick JS (2008) Chronic treatment with a type 5 phosphodiesterase inhibitor suppresses apoptosis of corporal smooth muscle by potentiating Akt signalling in a rat model of diabetic erectile dysfunction. Eur Urol 53:1282-1288

26. Bannowsky A, Schulze H, van der Horst C, Hautmann S, Jünemann KP (2008) Recovery of erectile function after nerve-sparing radical prostatectomy: improvement with nightly low-dose sildenafil. BJU Int 101:1279-1283

27. Briganti A, Di Trapani E, Abdollah F et al (2012) Choosing the best candidates for penile rehabilitation after bilateral nerve-sparing radical prostatectomy. J Sex Med 9:608-617

28. Castiglione F, Nini A, Briganti A (2014) Penile rehabilitation with phosphodiesterase type 5 inhibitors after nerve-sparing radical prostatectomy: are we targeting the right patients? Eur Urol 65:673-674

29. Briganti A, Gallina A, Suardi N et al (2010) Predicting erectile function recovery after bilateral nerve sparing radical prostatectomy: a proposal of a novel preoperative risk stratification. J Sex Med 7:2521-2531 